\title{
Free energy of the concerted-exchange mechanism for self-diffusions in silicon
}

\section{Citation}

Antonelli, A., S. Ismail-Beigi, Efthimios Kaxiras, and K. C. Pandey. 1996. "Free Energy of the Concerted-Exchange Mechanism for Self-Diffusion in Silicon." Physical Review B 53 (3): 131014. https://doi.org/10.1103/physrevb.53.1310.

\section{Permanent link}

http://nrs.harvard.edu/urn-3:HUL.InstRepos:41384072

\section{Terms of Use}

This article was downloaded from Harvard University's DASH repository, and is made available under the terms and conditions applicable to Other Posted Material, as set forth at http:// nrs.harvard.edu/urn-3:HUL.InstRepos:dash.current.terms-of-use\#LAA

\section{Share Your Story}

The Harvard community has made this article openly available.

Please share how this access benefits you. Submit a story.

Accessibility 


\title{
Free energy of the concerted-exchange mechanism for self-diffusion in silicon
}

\author{
A. Antonelli \\ Instituto de Física Gleb Wataghin, Universidade Estadual de Campinas, Unicamp, \\ 13083-970 Campinas, São Paulo, Brazil \\ S. Ismail-Beigi* and Efthimios Kaxiras \\ Department of Physics and Division of Applied Sciences, Harvard University, Cambridge, Massachusetts 02138 \\ K. C. Pandey \\ IBM Research Division, T. J. Watson Research Center, Yorktown Heights, New York 10598
}

(Received 8 August 1995 )

\begin{abstract}
The free energy of the concerted exchange mechanism for self-diffusion in silicon is estimated using the thermodynamic integration method and Monte Carlo (MC) simulations with an interatomic potential fitted to reproduce local-density-approximation calculations. Anharmonicity and relaxation are fully taken into account in the calculations, since the phase space is extensively explored by the MC simulations. The results indicate that the concerted exchange mechanism can have a significant contribution to the self-diffusion constant in silicon.
\end{abstract}

\section{INTRODUCTION}

Atomic diffusion in solids plays a very important role for various physical properties. In semiconductors, atomic diffusion is crucial for controlling doping, interfacial mixing, etc., which affect their electronic and optical properties. Silicon is by far the most extensively studied semiconductor. Nevertheless, our understanding of self-diffusion in silicon at the microscopic level remains an elusive issue. Since silicon is a covalent solid, it was expected that self-diffusion could only be mediated by native defects, such as vacancies and interstitials. Early theoretical studies ${ }^{1}$ found an activation enthalpy for defect mechanisms to be in the range $3.5-4.5 \mathrm{eV}$, in reasonable agreement with experimental data, which lie between 4.1 and $5.1 \mathrm{eV}^{2}$ Pandey has proposed a different mechanism, the concerted exchange $(\mathrm{CE})$, in which, two neighboring atoms exchange positions along a specific path, without the intervention of a native defect. ${ }^{3}$ The two atoms that are exchanging positions in the CE mechanism execute a complex motion in three-dimensional space in order to avoid large energy barriers. Along this path, bonds between the exchanging atoms and their nearest neighbors are successively broken and reformed. Figure 1 shows the exchanging atoms in their equilibrium positions and at the saddle-point configuration. The calculated activation enthalpy of $4.3 \mathrm{eV}$ for this mechanism ${ }^{3}$ is similar to those of the defect mechanisms and lies in the range of the experimental data.

From the above discussion it is evident that, based solely on the enthalpy, it is not possible to determine the existence of a dominant microscopic mechanism responsible for selfdiffusion in silicon. The experimental data show that the entropy contribution to the activation free energy for selfdiffusion is substantial, approximately $7 k_{B}$ to $9 k_{B} .^{2}$ Thus, since these microscopic mechanisms are very different in nature, theoretical estimates of the free energy and entropy could shed some light on the issue of the existence of a dominant self-diffusion mechanism. Quantities such as inter- nal energy and enthalpy are functions of the positions and velocities of the atoms, and can be easily determined from the calculations. The free energy and the entropy, on the other hand, are much more difficult to determine, because they depend on the total phase-space volume accessible to the system. ${ }^{4}$ Estimates of the activation entropy for the defect mechanisms were $\sim 1 k_{B} \cdot{ }^{5}$ Pandey and Kaxiras, ${ }^{6}$ applying classical transition-rate theory ${ }^{7}$ to the ideal energy surface (without relaxation) of the $\mathrm{CE}$ mechanism, obtained a lower bound for the entropy of this process of $3.3 k_{B}$. Subsequently, the same authors 8 included full relaxation of the energy surface, as well as contributions of vibrations within the harmonic approximation, yielding an activation entropy of $6.3 k_{B}$, which accounts for a substantial part of the experimental data. More recently, Blöchl et al., ${ }^{9}$ using parameterfree molecular-dynamics simulations, studied the defect mediated mechanisms for self-diffusion and found that the interstitialcy mechanism yields a diffusion coefficient very close to experiment, whereas the vacancy mechanism yields a diffusion coefficient 3 orders of magnitude smaller.

Since in the previous estimates of the activation entropy of the CE mechanism the entire phase-space available for the system was not completely explored, it would seem appropriate to readdress the question using a different methodology. Here we provide an accurate determination of the activation free energy for the $\mathrm{CE}$ mechanism using the

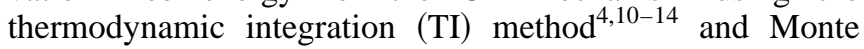
Carlo (MC) simulations to generate averages in the canonical ensemble. The TI method, initially proposed by Kirkwood, ${ }^{10}$ has been widely used over the years for a variety of problems such as vacancies in rare-gas solids, ${ }^{11}$ phase transitions, ${ }^{4,12}$ diffusion of impurities in $\mathrm{Si}^{13}$ extended defects in metallic alloys,${ }^{14}$ etc. In the following we describe the method and the approximations used in the calculations.

\section{SIMULATION METHOD}

In our calculations, the interaction between $\mathrm{Si}$ atoms was modeled using an effective two- and three-body potential, ${ }^{15}$ 
(a)

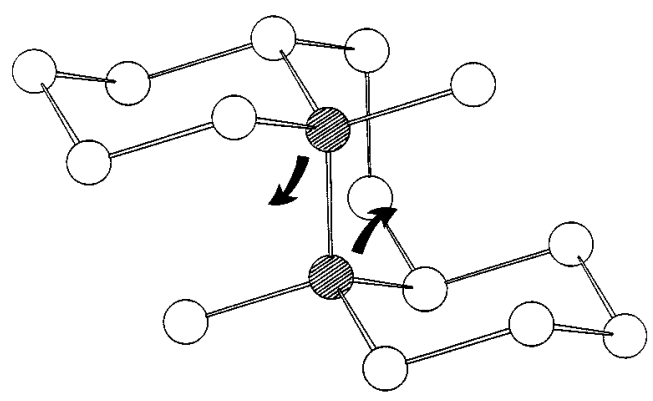

(b)

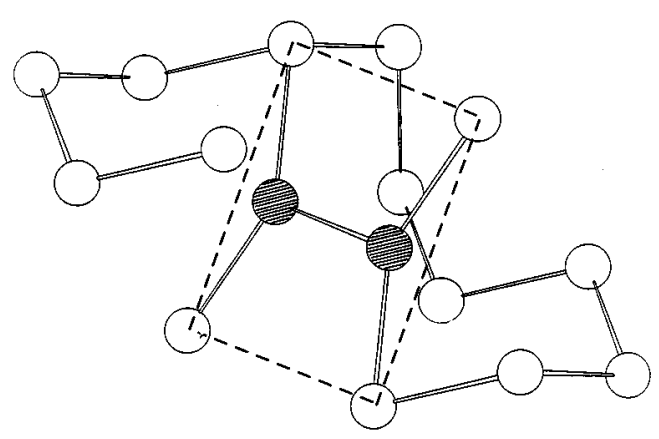

FIG. 1. Representation of the neighborhood of the CE mechanism in (a) the perfect crystal and (b) the saddle-point configurations. The atoms exchanging sites are shown shaded. In (a) the arrows indicate the motion of the atoms from the equilibrium toward the saddle-point point configuration. In (b) the dashed lines indicate the plane where the two atoms at the saddle-point are restricted.

which is based on a large database obtained from local density approximation (LDA) calculations. This potential reproduces the equilibrium lattice constant, the bulk modulus, and the cohesive energy of bulk $\mathrm{Si}$, as well as the LDA results for the energy along two paths in the CE energy landscape: the migration path and a path perpendicular to the migration path, passing through the saddle point. These two paths represent the most important features of the total-energy landscape. The migration path is obviously important because it contains the saddle point configuration. The path perpendicular to it is also important, because its curvature at the saddlepoint is related to the activation entropy within the classical transition-rate theory. ${ }^{7}$ Tests of this potential revealed that it also provides an excellent description of the fully relaxed saddle-point configuration, without having fitted it. Thus, it should be reliable for the type of calculations reported here.

The constant volume Monte Carlo simulations were performed using a supercell geometry and periodic boundary conditions to simulate the infinite crystal. The Monte Carlo method was implemented according to the Metropolis algorithm. Typically, $10^{4} \mathrm{MC}$ steps were used to achieve equilibration and averages were taken from samples with $5 \times 10^{4}$ MC steps. The Helmholtz free energy difference between two states with energy $E_{0}$ and $E_{0}+E_{1}$ is given in the TI method by

$$
\Delta F=\int_{0}^{1}\left\langle\frac{\partial E_{\lambda}}{\partial \lambda}\right\rangle d \lambda
$$

where $E_{\lambda}=E_{0}+\lambda E_{1}$ and \langle\rangle stands for the average in the canonical ensemble:

$$
\langle A\rangle=\int[d X] A e^{-E_{\lambda} / k_{B} T},
$$

where $\int[d X]$ represents an integral over the entire phase space available for the system. The energy $E_{\lambda}$ does not necessarily represent a physical system for $0<\lambda<1$. In most cases, the kinetic energy remains the same as $\lambda$ varies, since the overall number of particles in the system remains the same. Therefore, the kinetic energy does not contribute to the change in the free energy. Since this is the case here, we will not consider the kinetic energy in our calculations.

In order to calculate the activation free energy of the $\mathrm{CE}$ mechanism we considered the following potential energy, for a supercell of $N$ atoms:

$$
E_{\lambda}=E_{0}+\lambda \varepsilon_{1}+(1-\lambda) \varepsilon_{2},
$$

where $E_{0}$ includes the potential energy of all the atoms in the cell, except for those that are exchanging sites (a total of $N-2$ atoms), $\varepsilon_{1}$ is the potential energy of the two exchanging atoms constrained to vibrate around the saddle-point configuration, and $\varepsilon_{2}$ is the potential energy of the two exchanging atoms vibrating at the perfect crystal sites. During the simulation, care must be taken to keep the two exchanging atoms on the saddle-point surface, ${ }^{8}$ since otherwise the atoms would depart from the saddle-point configuration and move toward the perfect crystal geometry. In terms of the angular coordinates defined by Pandey to describe the CE mechanism, ${ }^{3}$ the following restriction is necessary near the saddle-point configuration: $\theta=-\phi+2 \pi / 3$. This constrains one degree of freedom in the system. To achieve the desired effect of keeping the two atoms on the saddle-point surface, an additional degree of freedom must be constrained; to this end, it suffices to constrain the center of mass of the two exchanging atoms to lie on the saddle-point surface, which near the saddle-point configuration coincides with the plane defined by their four neighbors [see Fig. 1(b)].

It should be pointed out that the two pairs of exchanging atoms at the perfect crystal configuration and at the saddlepoint configuration do not interact with each other, but they do interact with all the other atoms in the cell. In other words, for $\lambda=0$ one has $N$ atoms oscillating around the perfect crystal positions plus two atoms constrained at the saddle-point surface but decoupled from the rest of the system. For $\lambda=1$, there are $N-2$ atoms atoms vibrating around the perfect crystal positions interacting with two atoms constrained at the saddle-point plane, plus two atoms oscillating around the ideal crystal positions but decoupled from the rest of the system. The atoms that are decoupled from the rest of the system are confined to a sphere, in order to prevent them from wandering freely throughout the system. This can be easily implemented in the MC simulations by not accepting moves that take the atoms out of the spheres. The spheres, whose radius is taken to be approximately the amplitude of the atomic vibrations at each temperature, are centered at the positions of the atoms for the perfect crystal and the saddlepoint configurations. The integral in Eq. (1) is evaluated numerically using the Gauss-Legendre (GL) quadrature method. Initially, a 54-atom supercell and a 10-point GL 


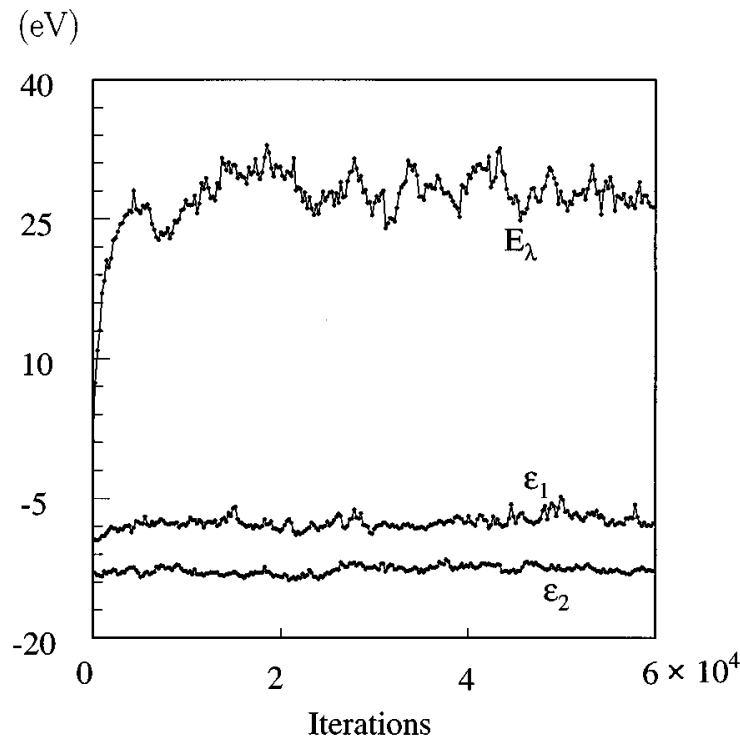

(a)

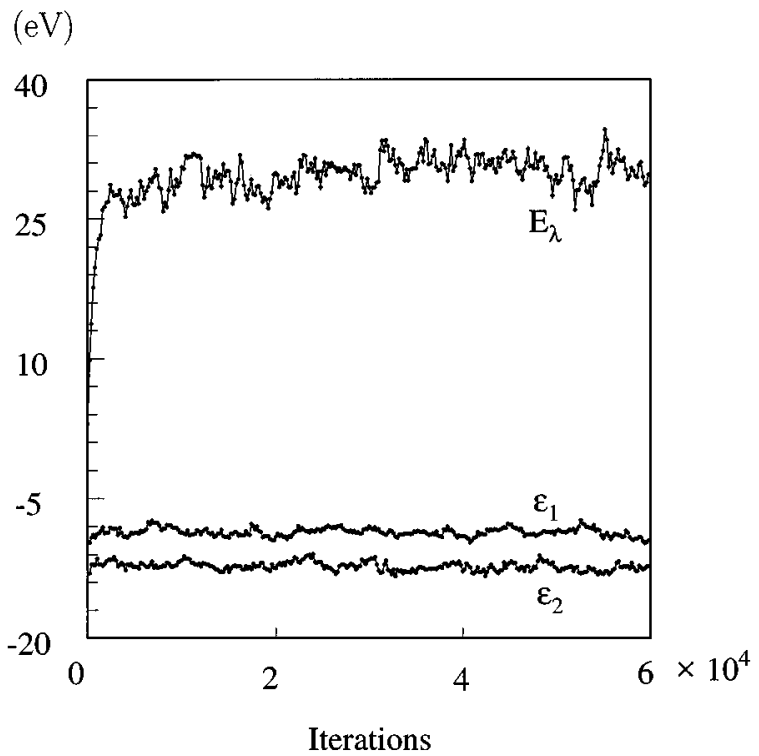

(b)

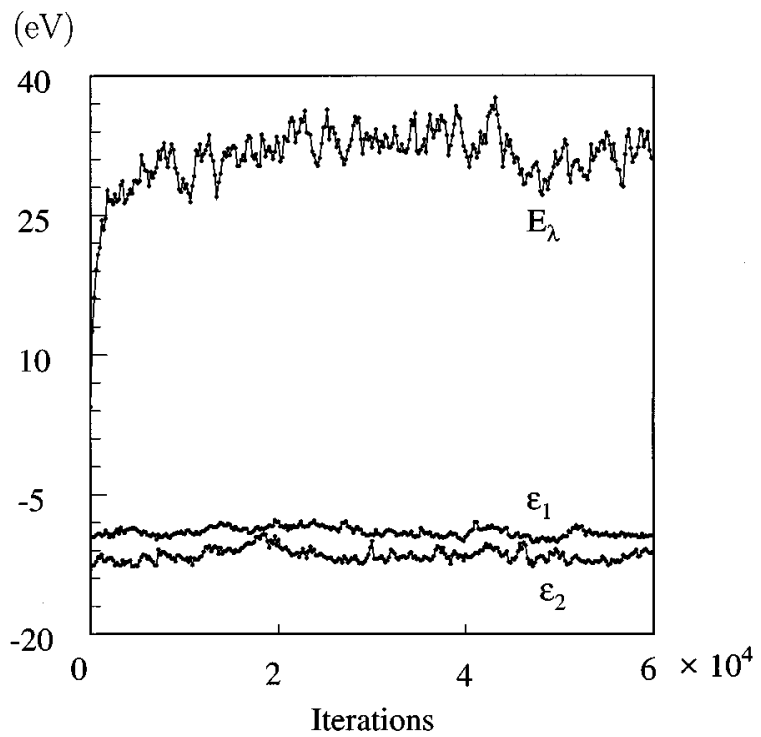

(c)

FIG. 2. Evolution of the potential energy components $\varepsilon_{1}, \varepsilon_{2}$, and the total potential energy $E_{\lambda}$ (see text) for (a) the lowest $(\lambda=$ $0.046910078)$, (b) the middle $(\lambda=0.5)$, (c) the largest $(\lambda=0.953089923)$, values of the parameter $\lambda$ of a 5-point GL quadrature, at a temperature $T=1500 \mathrm{~K}$. Energies are shown at intervals of $200 \mathrm{MC}$ steps. The energy of the 128 -atom supercell at $0 \mathrm{~K}$ is taken as the zero of the potential energy.

quadrature were used. The integrand in Eq. (1) turned out to be a very smooth function of $\lambda$. This test indicated that a GL quadrature with fewer points might be used without introducing substantial numerical error. By reducing the number of points in the GL quadrature we were able to increase significantly the size of the supercell. The results presented here were obtained using a 128-atom supercell and a 5-point GL quadrature.

\section{RESULTS}

Figure 2 shows the evolution of the components of the potential energy $\varepsilon_{1}$ and $\varepsilon_{2}$, as well as the total potential energy $E_{\lambda}$ during the MC simulation for three values of $\lambda$ at a temperature of $1500 \mathrm{~K}$. This figure contains simulation results for the smallest and largest values of $\lambda$ in the 5-point GL quadrature, as well as for the middle-point value. It is important to note that even for the smallest and largest $\lambda$ values, the extreme cases when there is a pair of particles almost decoupled from the rest of the system, the simulations are quite stable. This is the reason why the thermodynamic average in Eq. (1), which involves an integral of the quantity

$$
\frac{\partial E_{\lambda}}{\partial \lambda}=\varepsilon_{1}-\varepsilon_{2}
$$

is a smooth function of $\lambda$. As expected, however, the fluctuations are more pronounced for the smallest and largest $\lambda$ 
values. It should be noted that although the derivative in (4) does not depend explicitly on $\lambda$, the canonical average of it does depend on $\lambda$, since the atomic configurations during the simulations are generated using the potential energy $E_{\lambda}$ [see Eq. (2)]. Another interesting feature is that while $\lambda$ varies from 0 to 1 , the average of $\varepsilon_{1}$ remains approximately the same. On the other hand, the average of $\varepsilon_{2}$ increases with $\lambda$, that is the energy of the pair of atoms vibrating at the perfect crystal sites increases as they are decoupled from the rest of the system.

We can now compare our results with the experimental self-diffusion data. At high temperatures, the diffusion coefficient can be written as ${ }^{16}$

$$
D=D_{0} e^{-\Delta H / k_{B} T},
$$

where $D_{0}$ is the pre-exponential factor and $\Delta H$ is the activation enthalpy. In the case of the $\mathrm{CE}$ mechanism, the activation enthalpy is just the difference in enthalpy between the saddle point and the perfect crystal configurations. The preexponential factor is given by

$$
D_{0}=\frac{1}{6} f z a^{2} \nu e^{\Delta S / k_{B}},
$$

The factor $f$ is the correlation factor between consecutive jumps, which is equal to 1 for self-diffusion ocurring through exchange events. ${ }^{17}$ The coordination number $z$ in the case of a diamond lattice is 4 . The jump distance, $a$, is the interatomic distance in silicon. The factor $\nu=\sqrt{k_{B} T / 2 \pi \mu a^{2}}$ is the attempt frequency for the $\mathrm{CE}$ mechanism, where $\mu=m_{\mathrm{Si}} / 2$. The activation entropy $\Delta S$ is given by the sum of two terms,

$$
\Delta S=\Delta S_{\text {conf }}+\Delta S_{\text {vib }}
$$

where $\Delta S_{\text {conf }}=k_{B} \ln 6$, since there are 6 equivalent paths for the CE to take place. The factor $\Delta S_{\text {vib }}$ accounts for the difference in vibrational entropy between the saddle point and the perfect crystal configurations. From Eqs. (5) and (6) the self-diffusion coefficient can be written as

$$
D=\frac{2}{3} a^{2} \nu e^{-\Delta G / k_{B} T},
$$

where $\Delta G=-T \Delta S+\Delta H$ is the overall activation Gibbs free energy of the CE mechanism.

We performed constant volume MC simulations, therefore, the TI procedure yields the Helmholtz activation free energy. First-principles calculations have shown that the activation energy of the $\mathrm{CE}$ mechanism is weakly affected by hydrostatic pressure. ${ }^{18}$ It is then reasonable to assume that the Helmholtz activation free energy and the Gibbs activation free energy for the $\mathrm{CE}$ mechanism are essentially the same. In Fig. 3, we depict the calculated self-diffusion coefficient for three values of the temperature, which can be directly compared with the experimental results (straight lines). The averages of the MC runs are obtained by using different samples that are spaced apart by different intervals. The error bars represent the standard deviation from the average values. The error bars increase with temperature due to larger fluctuations at higher temperature. Our present results indicate that the inclusion of more degrees of freedom in the calculation through the TI formalism increases the self-

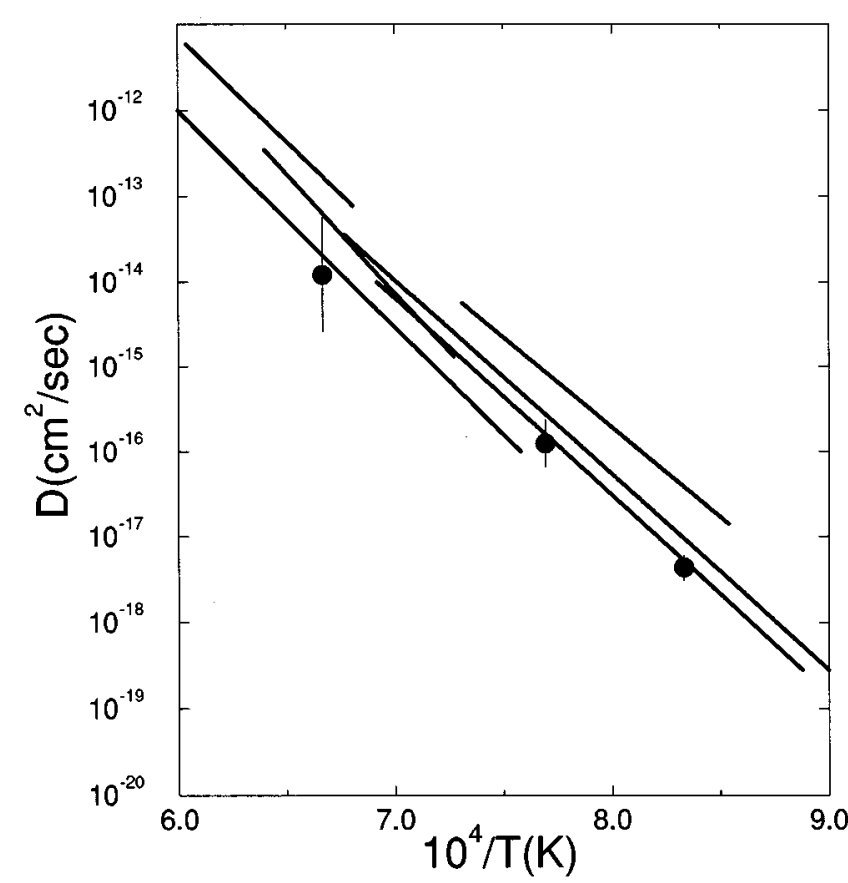

FIG. 3. Self-diffusion coefficient in silicon: calculated from CE mechanism (dots) and measured by experiment (straight lines), see Ref. 2.

diffusion coefficient for the CE mechanism, when compared with previous estimates. These results are in reasonable agreement with the experimental data, when the scattering in the latter is taken into account. Recalling that $\Delta F=-T \Delta S+\Delta E$, where $\Delta E$ is the variation in the internal energy, we are able extract from the present results the activation entropy $\Delta S$ for the $\mathrm{CE}$ mechanism. We obtained $7.8 \pm 0.4 k_{B}, 8.3 \pm 0.7 k_{B}$, and $8.1 \pm 1.5 k_{B}$ at 1200,1300 , and $1500 \mathrm{~K}$, respectively. Our findings for the entropy are in the experimental range of $7 k_{B}-9 k_{B}$.

\section{CONCLUSIONS}

We have estimated the activation free energy for the CE mechanism for self-diffusion in silicon in a wide range of temperatures, using the TI method and MC simulations with an interatomic potential fitted to reproduce the most important energy paths, calculated from first principles. The TI method allows for an efficient sampling of the phase space. The self-diffusion coefficient obtained from these estimates of free energy is in reasonable agreement with experimental measurements. As expected, the inclusion of more degrees of freedom brought the calculated values for the self-diffusion coefficient closer to the experimental results than those obtained in previous calculations. ${ }^{6,8}$ From these results, it seems that the CE mechanism can account for a substantial part of self-diffusion in silicon.

The present results, taken together with the free energies for the defect mechanisms, ${ }^{2,9}$ suggest that the expectation that the dominant mechanism for self-diffusion could be identified from the entropic contribution, has not been fulfilled. Given the considerable error bars involved in all the calculations of the diffusion constant (error bars of 2-3 orders of magnitude in the defect-mediated mechanisms ${ }^{9}$ and 
of 1-2 orders of magnitude in the present defect-free mechanism), we conclude that all mechanisms may be operative, since they yield self-diffusion coefficients in a range of 1 to 2 orders of magnitude, which is comparable to the uncertainty in experimental measurements. In light of this conclusion, we expect that the mechanism by which impurities diffuse will depend entirely on the nature of the impurity. It seems clear now that some additional experimental probe is required to test for a possible dominant microscopic mechanism among those proposed for self-diffusion in silicon. One possibility is to use hydrostatic pressure and/or anisotropic stress to probe the relative importance of the various possible mechanisms of self-diffusion in Si. A study by Aziz et al., ${ }^{19}$ using hydrostatic pressure, has shown that self-diffusion increases with pressure. This experiment was performed at a single temperature, and therefore, does not allow the separa- tion of the relative changes in the activation enthalpy and in the pre-exponential factor. Further experimental work of this type could provide valuable information on the issue of the self-diffusion mechanism in $\mathrm{Si}$.

\section{ACKNOWLEDGMENTS}

One of us (A.A.) wishes to thank Harvard University for its hospitality during the course of this work, as well as to acknowledge partial support from the Brazilian funding agencies Fundo de Apoio ao Ensino e à Pesquisa (FAEP/ UNICAMP) and Conselho Nacional de Desenvolvimento Científico e Tecnológico (CNPq). This work was supported in part by Harvard's Materials Science and Engineering Center, which is funded by NSF.
*Present address: Department of Physics, MIT, Cambridge, MA 02139.

${ }^{1}$ Y. Bar-Yam and J. D. Joannopulos, Phys. Rev. Lett. 52, 1129 (1984); Phys. Rev. B 30, 2216 (1984); R. Car, P. J. Kelly, A. Oshiyama, and S. T. Pantelides, Phys. Rev. Lett. 52, 1814 (1984); Phys. Rev. B 30, 2260 (1984); G. A. Baraff and M. Schlüter, ibid. 30, 3460 (1984).

${ }^{2}$ W. Frank, U. Gösele, H. Mehrer, and A. Seeger, in Diffusion in Crystalline Solids, edited by G. E. Murch and A. S. Nowick (Academic, Orlando, FL, 1985), p. 64.

${ }^{3}$ K. C. Pandey, Phys. Rev. Lett. 57, 2287 (1986).

${ }^{4}$ D. Frenkel, in Molecular Dynamics of Statistical-Mechanical Systems, Proceedings of the International School of Physics "Enrico Fermi," Course XCVII, edited by G. Ciccotti and W. G. Hoover (North-Holland, Amsterdam, 1986), p. 151.

${ }^{5}$ Y. Bar-Yam and J. D. Joannopoulos, in Microscopic Identification of Electronic Defects in Semiconductors, edited by N. M. Johnson, S. G. Bishop, and G. D. Watkins, MRS Symposia Proceedings Vol. 46 (Materials Research Society, Pittsburgh, 1985).

${ }^{6}$ K. C. Pandey and E. Kaxiras, Phys. Rev. Lett. 66, 915 (1991).

${ }^{7}$ G. H. Vineyard, J. Phys. Chem. Solids 3, 121 (1957).

${ }^{8}$ E. Kaxiras and K. C. Pandey, Phys. Rev. B 47, 1659 (1993).
${ }^{9}$ P. E. Blöchl, E. Smargiassi, R. Car, D. B. Laks, W. Andreoni, and S. T. Pantelides, Phys. Rev. Lett. 70, 2435 (1993).

${ }^{10}$ J. G. Kirkwood, J. Chem. Phys. 3, 300 (1935).

${ }^{11}$ C. H. Bennett, in Diffusion in Solids, edited by A. S. Nowick and J. J. Burton (Academic, New York, 1975), p. 74.

${ }^{12}$ J. Q. Broughton and X. P. Li, Phys. Rev. B 35, 9120 (1987).

${ }^{13}$ V. Milman, M. C. Payne, V. Heine, R. J. Needs, J. S. Lin, and M. H. Lee, Phys. Rev. Lett. 70, 2928 (1993).

${ }^{14}$ A. J. Skinner, J. V. Lill, and J. Q. Broughton, Modelling Simul. Mater. Sci. Eng. 3, 359 (1995).

${ }^{15}$ E. Kaxiras and K. C. Pandey, Phys. Rev. B 38, 12376 (1988).

${ }^{16}$ W. M. Franklin, in Diffusion in Solids, edited by A. S. Nowick, and J. J. Burton (Academic, New York, 1975).

${ }^{17}$ A. D. LeClaire in Diffusion Processes, edited by J. N. Sherwood, A. V. Chadwick, W. M. Muir, and F. L. Swinton (Gordon and Breach, London, 1971).

${ }^{18}$ A. Antonelli and J. Bernholc, Phys. Rev. B 40, 10643 (1989).

${ }^{19}$ M. J. Aziz, E. Nygren, W. H. Christie, C. W. White, and D. Turnbull, in Impurity Diffusion and Gettering in Silicon, edited by R. B. Fair, C. W. Pearce, and J. Washburn, MRS Symposia Proceedings Vol. 36 (Materials Research Society, Pittsburgh, 1985), p. 101. 